\title{
Lower limb and associated injuries in frontal-impact road traffic collisions.
}

\author{
Mohannad B. Ammori ${ }^{1,2}$, Hani O. Eid², Fikri M. Abu-Zidan ${ }^{2}$
}

1. Royal Bolton Hospital, Farnworth, Bolton, United Kingdom

2. Department of Surgery, College of Medicine and Health Sciences, United Arab Emirates University, Al-Ain, UAE

\begin{abstract} and abdominal regions in frontal-impact road traffic collisions. spared from a greater severity of head injury.

Keywords: Frontal-impact, road traffic collision, lower limb injury.

DOI: http://dx.doi.org/10.4314/ahs.v16i1.40 2016;16(1): 306-310. bttp://dx.doi.org/10.4314/abs.v16i1.40

\section{Introduction}

An analysis of the biomechanics of a road traffic collision can provide clues to establish the diagnosis of occult injuries $^{1,2}$. An appreciation of the mechanisms of injury and the relationship between the injuries sustained is imperative to guiding the clinical suspicion of the physician towards certain injuries when conducting the primary and secondary survey. An example of this is the raised probability of a solid organ injury in the presence of a concomitant fracture to the rib and the pelvis ${ }^{3}$.
\end{abstract}

Objectives: To study the relationship between severity of injury of the lower limb and severity of injury of the head, thoracic,

Methods: Consecutive hospitalised trauma patients who were involved in a frontal road traffic collision were prospectively studied over 18 months. Patients with at least one Abbreviated Injury Scale (AIS) $\geq 3$ or AIS 2 injuries within two AIS body regions were included. Patients were divided into two groups depending on the severity of injury to the head, chest or abdomen. Low severity group had an AIS $<2$ and high severity group had an AIS $\geq 2$. Backward likelihood logistic regression models were used to define significant factors affecting the severity of head, chest or abdominal injuries.

Results: Eighty-five patients were studied. The backward likelihood logistic regression model defining independent factors affecting severity of head injuries was highly significant $(p=0.01$, nagelkerke $r$ square $=0.1)$ severity of lower limb injuries was the only significant factor $(\mathrm{p}=0.013)$ having a negative correlation with head injury (Odds ratio of 0.64 (95\% CI: 0.45-0.91).

Conclusion: Occupants who sustain a greater severity of injury to the lower limb in a frontal-impact collision are likely to be

Cite as: Ammori MB, Eid HO, Abu-Zidan FM. Lower limb and associated injuries in frontal-impact road traffic collisions. Afri Health Sci.
Correspondling author:
Fikri M. Abu-Zidan,
Acute Care Surgeon,
Point-of-Care Sonographer,
and Statistical Consultant
Department of Surgery, College of Medicine and
Health Sciences,
UAE University, Al-Ain, United Arab Emirates
Tel 0097137137579
Fax: 0097137672067
E-mail: fabuzidan@uaeu.ac.ae

In a frontal-impact collision, there is a sudden deceleration of the vehicle. The force of the initial collision is frequently transmitted throughout the lower limbs ${ }^{4}$. Furthermore, the knee may collide with the dashboard whilst in flexion, which could result in a posterior hip dislocation $^{5}$. Lee et al reported that restrained occupants who sustained an injury to the knee, thigh or hip were four times less likely to suffer from a serious intra-abdominal injury in a frontal-impact road traffic collision ${ }^{6}$. Although the advantages of the safety belt are well-established ${ }^{7,8}$, the relationship between lower limb injuries and other body regions is less well understood.

We hypothesized that, in a frontal-impact collision, a greater severity of injury to the lower limb is preventative of a compressive load and the consequential severity of injury to the head, thoracic, and abdominal region. We aimed to study the relationship between the severity of injury to the lower limb and the severity of injury to the head, thoracic, and abdominal regions in frontal-impact road traffic collisions. 


\section{Patients and methods}

Between April 2006 and October 2007, the data of consecutive patients who sustained injuries, as a consequence of a frontal-impact road traffic collision, requiring admission, or those who died after arrival to the Emergency Department in Al-Ain or Tawam Hospital, were prospectively collected. Al-Ain Hospital and Tawam Hospital are the two major trauma centres of Al-Ain, which is the fourth largest city in the United Arab Emirates with a population of around 500,000. The patients or their caregivers signed a consent form for the acquisition of data for research purposes. A unique case number was used to identify each patient and the data entered into the database was anonymous. The patients of the present study are a subset of the cohort used for previously published research into other elements of the injuries sustained in road traffic collisions ${ }^{7}$. This study was approved by The Local Ethics Committee of Al-Ain Health district area (RECA/02/44) and was performed in accordance with the ethical standards laid down in the 1964 Declaration of Helsinki and its later amendments.

The information included patient demographics such as age and gender, the patient's position within the vehicle, ejection from the vehicle, and descriptions of the injuries sustained. The injuries sustained were classified according to the Injury Severity Score (ISS) and the Abbreviated Injury Scale (AIS). Patients with at least one region with an AIS $\geq 3$ or AIS 2 injuries within two AIS body regions were selected for this study. Patients must have been travelling inside a vehicle and had a frontal-impact collision to be included. Pedestrians, cyclists and motorcyclists were excluded, as they would be subject to an alternative mechanism of injury.

For the purposes of this study, the AIS anatomical regions concerned were the lower extremities, the head, thorax, and abdomen. Severity of injury of each region was graded as follows: no injury (AIS $=0)$, minor (AIS =
1), moderate (AIS $=2$ ), serious (AIS $=3$ ), severe (AIS = 4), and critical (AIS = 5).

\section{Statistical analysis}

Data was expressed as median (range) or number (percentage) as appropriate. Patients were divided into two groups depending on the severity of injury to the head, chest or abdomen. Low severity group had an AIS $<2$ and high severity group had an AIS $\geq 2$. Backward likelihood logistic regression models were used to define significant factors affecting the severity of head, chest or abdominal injuries. The dependent outcome variable was the head, chest, or abdominal injury severity group (low or high severity group) while the independent variables were age, gender, usage of safety seatbelt, whether thrown from the vehicle, severity of lower limb injury (ordinal data), and position in the vehicle (driver, front seat passenger, or back seat passenger, categorical data). Significance was accepted at $\mathrm{p}<0.05$ in the model. We analysed the data using the software package SPSS 20 (Chicago, Illinois, USA).

\section{Results}

A total of 256 patients, who were vehicle occupants, were involved in frontal-impact collisions, 85 (33.2\%) of who met the inclusion criteria for the study. They had median (range) age of 26 (2-58) years and 65 (76.5\%) were male. The demographic details of the patients are summarised in Table 1.

The backward likelihood logistic regression model defining independent factors affecting severity of head injuries was highly significant ( $p=0.01$, Nagelkerke R Square $=0.1$ ) Severity of lower limb injuries was the only significant factor $(p=0.013)$ having a negative correlation with head injury (Estimate of - 0.44 and an odds ratio of 0.64). The studied models using the same variables did not predict severity of chest injury ( $p=0.13$, Nagelkerke $\mathrm{R}$ Square of 0.04 ) neither severity of abdominal injuries ( $p=0.11$, Nagelkerke R Square of 0.05). 


\section{Table 1: Demography of the studied hospitalized vehicle occupants $(n=85)$ of frontal-impact road traffic collisions}

\section{Variable}

Age (years)

Male / Female

Driver / Front seat passenger/ Back seat passenger45 (52.9 \%) /24 (28.2\%)/16 (18.8 \%)
$26(2-58)$

$65(76.5 \%) / 20(23.5 \%)$
Seatbelt usage

Ejected

Injury Severity Score

Mortality
$12(14.1 \%)$

$11(12.9 \%)$

$14(8-45)$

$4(4.7 \%)$

Categorical data are presented as number (percentage) and ordered and continuous data as median (range) as appropriate

\section{Discussion}

This study has demonstrated that occupants who sustained a greater severity of injury to the lower limb in a frontal-impact collision were often spared injuries of a greater severity to the head. We found no association between the severity of injury to the lower limb and the severity of injury to the thorax or the abdomen.

A frontal-impact collision is characterised by a sudden deceleration of the vehicle, and a subsequent deceleration of the vehicle occupant through the application of direct stresses to bodily regions. A direct axial compressive load may be applied to the lower limb, as this regularly sits as the most anterior component of the body of the seated occupant. Drivers are subjected to an additional compressive stress on the femur due to the application of their right foot on the brake pedal and the contraction of muscle when bracing. In the event that the force is sufficient to result in a fracture or dislocation, the lower limb would have absorbed a significant portion of the energy generated upon impact. Although sustaining injuries to the lower extremities is, of course, undesirable and unintentional to the vehicle occupant, it is not as life threatening as injuries to the head, chest or abdomen ${ }^{6}$.

In a cohort of occupants who wore safety belts, Lee et al reported a negative correlation between a knee, thigh or hip injury and serious intra-abdominal injuries. Lee et al attributed a serious intra-abdominal injury to the safety belt. The contradictory absence of an association between a fracture or dislocation of the lower limb and the severity of intra-abdominal injury in our study is supportive of Lee et al's findings $\mathrm{s}^{6}$ because the majority of vehicle occupants $(86.4 \%)$ in our study did not wear a safety belt. The absence of a safety belt alters the mechanism of injury. In a frontal-impact collision, the head, neck and trunk of an unrestrained occupant would travel at a greater velocity in comparison to the lower limb due to the distribution of body mass and the muscular contraction of the lower limb against the toe pan, which provides 
resistance to forward translation ${ }^{10}$. If a fracture or dislocation of the lower limb were not sustained, a greater compressive force would have probably acted against the head and thoracic region, as a consequence of impaction against the windscreen and the steering wheel.

\section{Limitations}

We acknowledge that our study failed to include patients who were pronounced dead prior to arrival at Al-Ain or Tawam Hospital. A natural selection bias must be considered in the interpretation of these results, as patients with a greater severity of thoracic or head injuries may have been pronounced dead at the scene or prior to arrival at the Emergency Department. However, it is important to note the application of the results of this study to those patients who arrive at the Emergency Department for assessment and management in the trauma bay.

The patient cohort for our study was relatively small. This was demonstrated by the large confidence interval of the odds ratio of the severity of the lower limb injury in the direct logistic regression model (Table 2). However, this data includes all patients who were admitted or died after arrival to the Emergency Department with injuries sustained in a frontal-impact road traffic collision over an 18 -month period in a city, with a population of around 500,000 .

\section{Table 2: Backward likelihood logistic regression model defining independent factors affecting severity of head injuries}

\begin{tabular}{|c|c|c|c|c|c|c|}
\hline Variable & Estimate & SE & Wald test & $P$ value & OR & $\begin{array}{ll}95 \% & \text { CI } \\
\text { OR } & \end{array}$ \\
\hline Severity of lower limb injury & $\begin{array}{c}-0.44 \\
\end{array}$ & 0.18 & 6.24 & 0.013 & 0.64 & $0.45-0.91$ \\
\hline Constant factor & 0.42 & 0.3 & 1.87 & 0.17 & 1.52 & \\
\hline
\end{tabular}

Odds Ratio (OR); Confidence Interval (CI)

Although an analysis of the effect of specific types of lower limb injury in predicting severity of injuries of other body regions was considered, this could not be undertaken due to the limited number of patients of this study. The logistic regression model was highly significant, but the $\mathrm{R}$ square value was low (0.1), which is indicative that the findings of the direct logistic regression model explain approximately $10 \%$ of the variation of the data.

\section{Conclusion}

An understanding of the mechanisms of vehicle occupant injury in frontal-impact collisions is useful in determining the association of injuries sustained in different body regions. Occupants who sustain a greater severity of injury to the lower limb in a frontal-impact collision are likely to be spared from a greater severity of head injury.

\section{Acknowledgements}

This study was supported by an interdisciplinary UAE University grant no. 02-078-1/4.

\section{Conflict of interest}

None declared by all authors.

\section{References}

1. American College of Surgeons. Biomechanics of Injury. In: American College of Surgeons (eds). Advanced Trauma Life Support for Doctors, 7th ed. Chicago, IL; 2004. P. 315-335. 
2. Mackay M. Engineering in accidents: vehicle design and injuries. Injury 1994; 25:615-21.

3. Al-Hassani A, Afifi I, Abdelrahman H, El-Menyar A, Almadani A, Recicar J, Al-Thani H, Maull K, Latifi R. Concurrent rib and pelvic fractures as an indicator of solid abdominal organ injury. Int J Surg. 2013;11:483-6.

4. Eid HO, Abu-Zidan FM. Biomechanics of road traffic collision injuries: a clinician's perspective. Singapore Med J 2007; 48:693-700

5. Moore KL, Dalley AF. Lower Limb. In: Clinically Oriented Anatomy. 5th ed, Lippincott Williams \& Wilkins, Philadelphia; 2006. P. 683.

6. Lee J, Conroy C, Coimbra R, Tominaga GT, Hoyt DB. Injury patterns in frontal crashes: the association between knee-thigh-hip (KTH) and serious intra-abdominal injury. Accid Anal Prev 2010; 42:50-55

7. Abu-Zidan FM, Abbas AK, Hefny AF, Eid HO, Grivna M. Effects of seat belt usage on injury pattern and outcome of vehicle occupants after road traffic collisions: prospective study. World J Surg 2012; 36:255-259

8. Abbas AK, Hefny AF, Abu-Zidan FM. Seatbelts and road traffic collision injuries. WorldJ Emerg Surg. 2011;6:18. 9.. Tencer AF, Kaufman R, Ryan K, Grossman DC, Henley BM, Mann F et al. Crash Injury Research and Engineering Network (CIREN). Femur fractures in relatively low speed frontal crashes: the possible role of muscle forces. Accid Anal Prev 2002; 34:1-11

10. De Leva P. Adjustments to Zatsiorsky-Seluyanov's Segment Inertia Parameters. J Biomechanics 1996; 29:12231230. 